\title{
Mechanism of Immediate Recurrences of Atrial Fibrillation After Restoration of Sinus Rhythm
}

\author{
AMAN CHUGH, MEHMET OZAYDIN, CHRISTOPH SCHARF, STEVE W.K. LAI, BURR HALL, \\ PETER CHEUNG, FRANK PELOSI, JR, BRADLEY P. KNIGHT, FRED MORADY, \\ and HAKAN ORAL
}

From the Division of Cardiology, Department of Internal Medicine, University of Michigan, Ann Arbor, Michigan

\begin{abstract}
CHUGH, A., ET AL.: Mechanism of Immediate Recurrences of Atrial Fibrillation After Restoration of Sinus Rhythm. An immediate recurrence of AF may occur after restoration of sinus rhythm. Although pulmonary vein $(P V)$ isolation has been shown to prevent immediate recurrence of $A F$, the specific trigger for immediate recurrence of $A F$ has not been described. In 89 consecutive patients (mean age $53 \pm 11$ years) who had sinus rhythm restored by spontaneous or transthoracic cardioversion in the course of a PV isolation procedure, electrograms recorded within $a P V$ and in the adjacent left atrium were analyzed to determine the mechanism of initiation of immediate recurrence of $A F$. Immediate recurrence of $A F$ was defined as a recurrence of $A F$ within 90 seconds after restoration of sinus rhythm. There were 124 episodes of immediate recurrence of $A F$ at a mean of $18 \pm 23$ seconds after cardioversion. Recordings within the $P V$ that triggered the immediate recurrence of $A F$ were available in $23(19 \%)$ of the 124 immediate recurrence of $A F$ episodes. Among these 23 episodes of immediate recurrence of $A F$, all (100\%) were triggered by a burst of $P V$ tachycardia $(P<0.001)$. The coupling interval and prematurity index (coupling interval/preceding sinus cycle length) of the premature depolarizations that did and did not trigger immediate recurrence of $A F$ were $246 \pm 67 \mathrm{~ms}$ and $0.30 \pm 0.11 \mathrm{vs} 378 \pm 117 \mathrm{~ms}$ and $0.49 \pm 0.16$, respectively $(P<0.01)$. Immediate recurrence of $A F$ was abolished by $P V$ isolation. The mechanism of immediate recurrence of $A F$ is a burst of $P V$ tachycardia, not a single premature depolarization. Immediate recurrence of $A F$ identifies patients with AF in whom the PVs may play a major role in the initiation of AF. (PACE 2004; 27:77-82)
\end{abstract}

atrial fibrillation, cardioversion, pulmonary vein

\section{Introduction}

An immediate recurrence of atrial fibrillation (AF) may occur after restoration of sinus rhythm in patients with $\mathrm{AF}^{1-4}$ Some studies have suggested that immediate recurrence of AF is triggered by premature depolarizations, and that the probability of immediate recurrence of AF is dependent on the prematurity of the ectopic beat. ${ }^{5,6} \mathrm{~A}$ more recent study demonstrated that pulmonary vein (PV) isolation consistently abolishes the immediate recurrence of $\mathrm{AF}^{4}$ However, the specific triggers of the immediate recurrence of AF have not been well described. The aim of this study was to determine the mechanism of initiation of the immediate recurrence of $\mathrm{AF}$ in patients with AF.

Supported in part by the Ellen and Robert Thompson Atrial Fibrillation Research Fund.

Address for reprints: Hakan Oral, M.D., Cardiology, TC B1 140, 1500 E. Medical Center Dr., Ann Arbor, MI 48109-0311. Fax: (734) 936-7026; e-mail: oralh@umich.edu

Received March 3, 2003; revised May 8, 2003; accepted May 29, 2003.

\section{Patients and Methods \\ Patient Characteristics}

The study included 89 consecutive patients who underwent PV isolation for paroxysmal $(\mathrm{n}=$ 73) or persistent $(n=16)$ AF. AF that had been present for $>1$ month without any intervening episodes of sinus rhythm and required cardioversion for restoration of sinus rhythm was considered persistent. There were 78 men and 11 women (mean age $53 \pm 11$ years). The mean left ventricular ejection fraction was $0.54 \pm 0.04$, and the mean left atrial diameter was $41 \pm 5 \mathrm{~mm}$. AF was first diagnosed $7 \pm 7$ years before the PV isolation procedure. Among the 89 patients, 78 had no structural heart disease and $11(12 \%)$ had structural heart disease (i.e., coronary artery disease $n=4$, valvular heart disease $\mathrm{n}=4$, and a nonischemic cardiomyopathy $\mathrm{n}=3$ ).

\section{Electrophysiological Study}

All patients provided informed, written consent. Antiarrhythmic drug therapy was discontinued at least 5 half-lives before the procedure, except for amiodarone, which was discontinued at least 4 weeks previously. A quadripolar electrode 
catheter (EP Technologies Inc., Mountain View, CA, USA) was inserted into a femoral vein and positioned in the coronary sinus. After transeptal catheterization, a decapolar ring catheter (Lasso, Biosense Webster, Inc., Diamond Bar, CA, USA) and a deflectable, quadripolar catheter (EP Technologies Inc.) with 2-5-2-mm interelectrode spacing and a 4-mm distal electrode were inserted into the left atrium. Systemic anticoagulation was achieved with intravenous heparin to maintain an activated clotting time of 250-350 seconds.

Bipolar intracardiac electrograms were filtered at band-pass settings of 30-500 $\mathrm{Hz}$ and were recorded digitally (EP MedSystems, Inc., West Berlin, NJ, USA). Atrial pacing was performed from the coronary sinus (EP MedSystems, Inc., Model EP-3 Clinical Stimulator).

\section{Study Protocol}

Among the 89 patients, $42(47 \%)$ were in AF and $47(53 \%)$ were in sinus rhythm at the onset of the procedure. In patients who presented in sinus rhythm, AF was induced by atrial pacing at a cycle length of 200-180 ms.

During AF, electrograms were recorded within each PV in sequence with the ring catheter. In a prior study, all episodes of immediate recurrence of AF were abolished by isolation of the PVs, indicating that the PVs are the source of triggers for immediate recurrence of $\mathrm{AF}^{4}$ Therefore, to characterize the trigger of immediate recurrence of $\mathrm{AF}$, the ring catheter was positioned within a PV during cardioversion. Because it was not feasible to place a ring catheter in each of the four PVs simultaneously, the catheter was positioned inside the left superior, right superior, or left inferior PV, within $5 \mathrm{~mm}$ of the ostium. In general, the ring catheter was positioned within the PV that had the most frequent bursts of rapid electrical activity consistent with a PV tachycardia. ${ }^{7}$ A quadripolar catheter was positioned in the left atrium, adjacent to the PV in which the ring catheter was positioned. To prevent catheter induced atrial depolarizations during cardioversion, firm contact of this catheter against the atrial wall was avoided.

Transthoracic cardioversion was performed after conscious sedation with midazolam and fentanyl. Adhesive electrodes were positioned on the anterior and posterior chest. Sinus rhythm was restored with a conventional defibrillator using monophasic shocks of 200-360 J (HewlettPackard, Andover, MA, USA). In 4 (4\%) of the 89 patients, AF converted to sinus rhythm spontaneously.

Immediate recurrence of $\mathrm{AF}$ was defined as an episode of AF that occurred within 90 seconds after transthoracic or spontaneous cardioversion (Fig. 1).

\section{Analysis of Intracardiac Electrograms}

Intracardiac electrograms were analyzed offline to determine the mechanism of immediate recurrence of AF. Premature depolarizations recorded within a PV were considered to have originated in that PV if they preceded the atrial electrogram recorded in the adjacent left atrium (Fig. 1). PV depolarizations that occurred simultaneous with or after the adjacent left atrial electrogram were considered to represent passive activation of the PV. A PV tachycardia was defined as a burst of repetitive electrical activity within a PV that preceded left atrial activation, and that had a shorter cycle length than at the adjacent left atrium, or that had cycle length oscillations that preceded changes in the left atrial cycle length (Fig. 1).

The coupling interval of premature depolarizations was defined as the interval from the prior sinus depolarization to the onset of the premature depolarization. The prematurity index was defined as the ratio of the coupling interval of the premature depolarization to the preceding sinus cycle length (Fig. 2).

\section{PV Isolation}

Electrical isolation of the PVs was performed by segmental ostial applications of conventional radiofrequency energy, guided by PV potentials, as described previously. ${ }^{4,8,9}$

\section{Statistical Analysis}

Continuous variables are expressed as mean \pm 1 SD. Continuous variables were compared with the Student's $t$-test. Categorical variables were compared by chi-square analysis or with the Fisher's exact test, as appropriate. A P value $<0.05$ indicated statistical significance.

\section{Results \\ Prevalence of Immediate Recurrence of AF}

Among the 89 patients, 50 (56\%) experienced 124 episodes of immediate recurrence of AF. Immediate recurrence of AF occurred a mean of $18 \pm$ 23 seconds after restoration of sinus rhythm. The prevalence of immediate recurrence of $\mathrm{AF}$ was similar in patients who had spontaneous $(65 \%)$ or pacing induced $\mathrm{AF}(56 \%, \mathrm{P}=0.4)$. Immediate recurrence of AF occurred after 17 of 67 episodes of spontaneous termination of AF (25\%) and after 107 of 192 successful transthoracic cardioversions $(56 \%, \mathrm{P}<0.01)$.

\section{Premature Depolarizations After Restoration of Sinus Rhythm}

In the 50 patients with an episode of immediate recurrence of $\mathrm{AF}$, there were 301 premature 


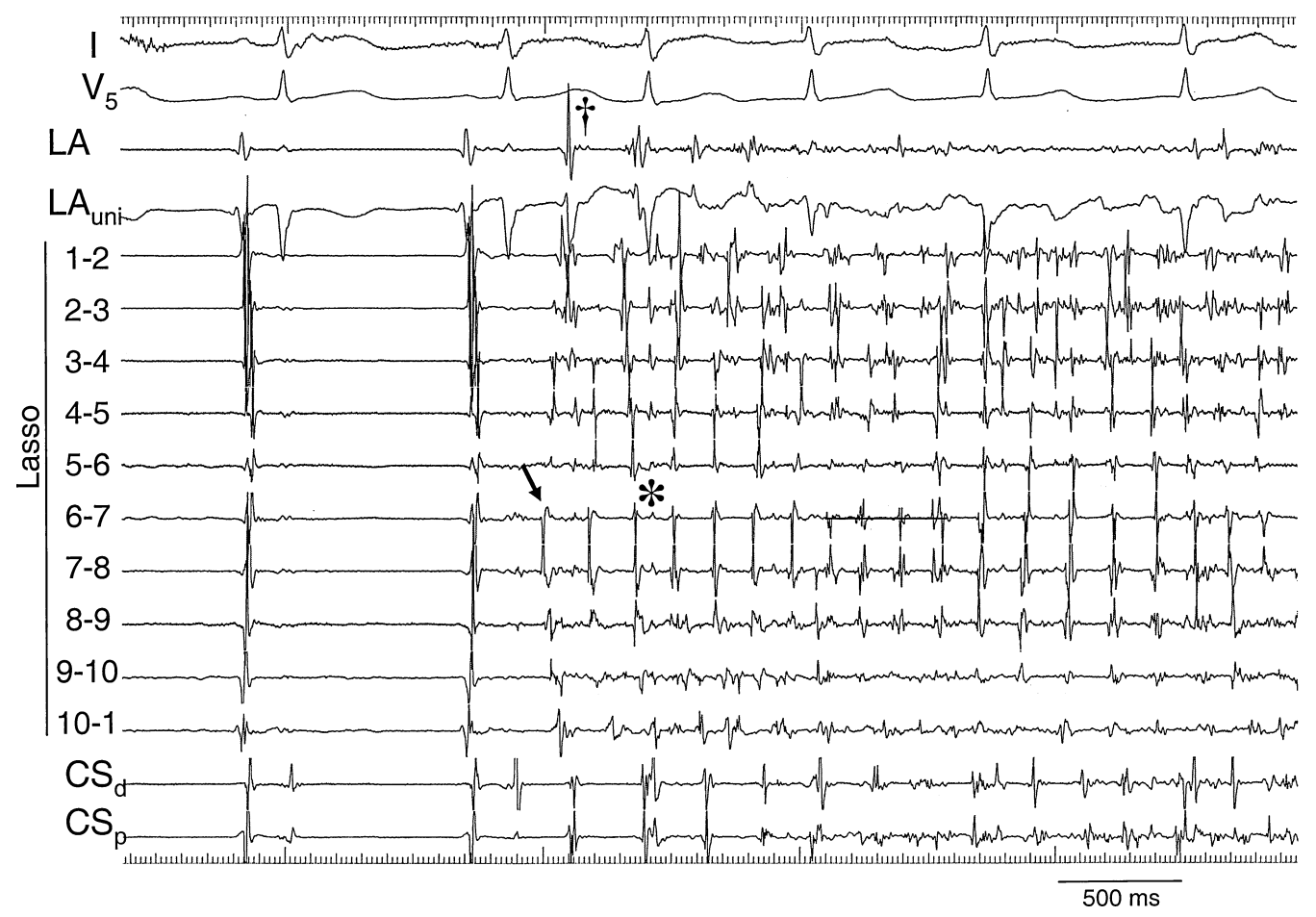

Figure 1. Pulmonary vein (PV) tachycardia and immediate recurrence of atrial fibrillation (AF) after transthoracic cardioversion. Shown are leads $I$, and $V_{5}$, bipolar, and unipolar recordings from the distal electrode pair of a mapping catheter positioned in the left atrium just outside the ostium of the left superior PV ( $\left.L A, L A_{\text {uni }}\right)$, a decapolar Lasso catheter positioned within the left superior $P V\left(L_{1-2}, \ldots, L_{10-1}\right)$, and distal and proximal electrode pairs of a coronary sinus catheter $\left(C S_{d}, C S_{p}\right)$. After transthoracic cardioversion, a PV tachycardia that has a mean cycle length of $125 \mathrm{~ms}$ (asterisk) triggers immediate recurrence of AF. The cycle length within the adjacent left atrium was $188 \mathrm{~ms}$. The first premature PV depolarization (arrow) precedes the left atrial electrogram (dagger) recorded just outside the PV ostium.

atrial depolarizations that occurred within 90 seconds after restoration of sinus rhythm. Among these 301 premature atrial depolarizations, a PV was documented to be the source of the depolarization(s) in 120 (40\%). Within the same PVs, 16 PV depolarizations that did not conduct to the left atrium also were recorded. The prematurity index of the first beat of the premature PV depolarizations that did and did not conduct to the left atrium were $0.45 \pm 16$ and $0.20 \pm 0.08$, respectively, $(\mathrm{P}<$ 0.01).

Premature PV depolarizations occurred at a mean of $26 \pm 23$ seconds after restoration of sinus rhythm. Among the 136 premature PV depolarizations, $84(61 \%)$ were single, $24(18 \%)$ were double, $5(4 \%)$ were triple, and $23(18 \%)$ occurred in the form of a PV tachycardia.

\section{Mechanisms of Initiation of Immediate Recurrence of AF}

Among the 124 episodes of immediate recurrence of $\mathrm{AF}$, the trigger was recorded by the ring catheter that was positioned within a PV in $23(19 \%)$. In all episodes $(100 \%)$, the trigger of immediate recurrence of AF was a burst of PV tachycardia. The mechanism of initiation of immediate recurrence of AF was by a PV tachycardia regardless of whether AF was paroxysmal of persistent.

Among these 23 episodes of immediate recurrence of $A F, 2$ (9\%) occurred in patients with persistent $\mathrm{AF}$ and 21 in patients with paroxysmal AF. During 23 episodes of immediate recurrence of AF recorded from a ring catheter, the catheter was positioned within the left superior PV in 16 (70\%), the right superior PV in $4(17 \%)$, and the left inferior PV in $3(13 \%)$ patients.

Among 84 single premature PV depolarizations that were recorded after cardioversion, none initiated an episode of immediate recurrence of AF. Among 23 bursts of PV tachycardia that were recorded after cardioversion, $23(100 \%)$ triggered an episode of immediate recurrence of AF (Fig. 2). The coupling interval and the prematurity index 
A

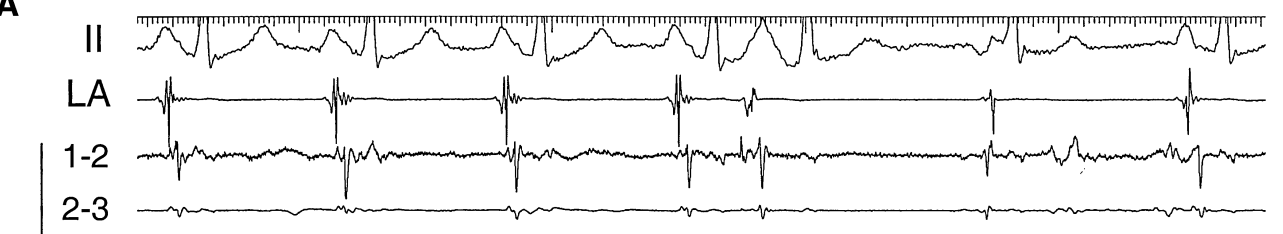

3-4
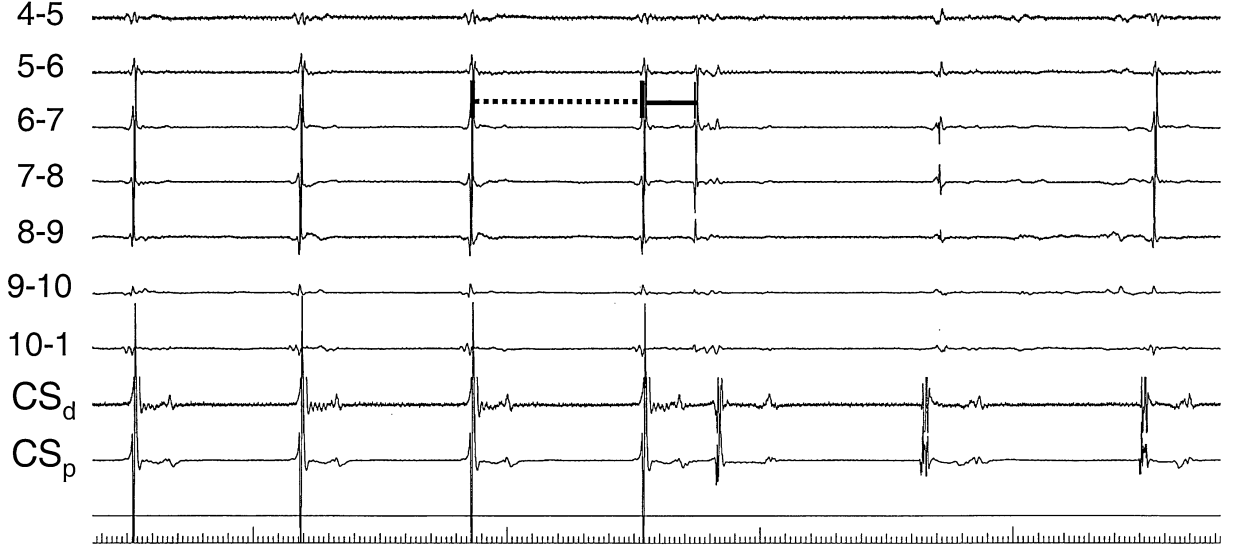

$500 \mathrm{~ms}$

\section{B}
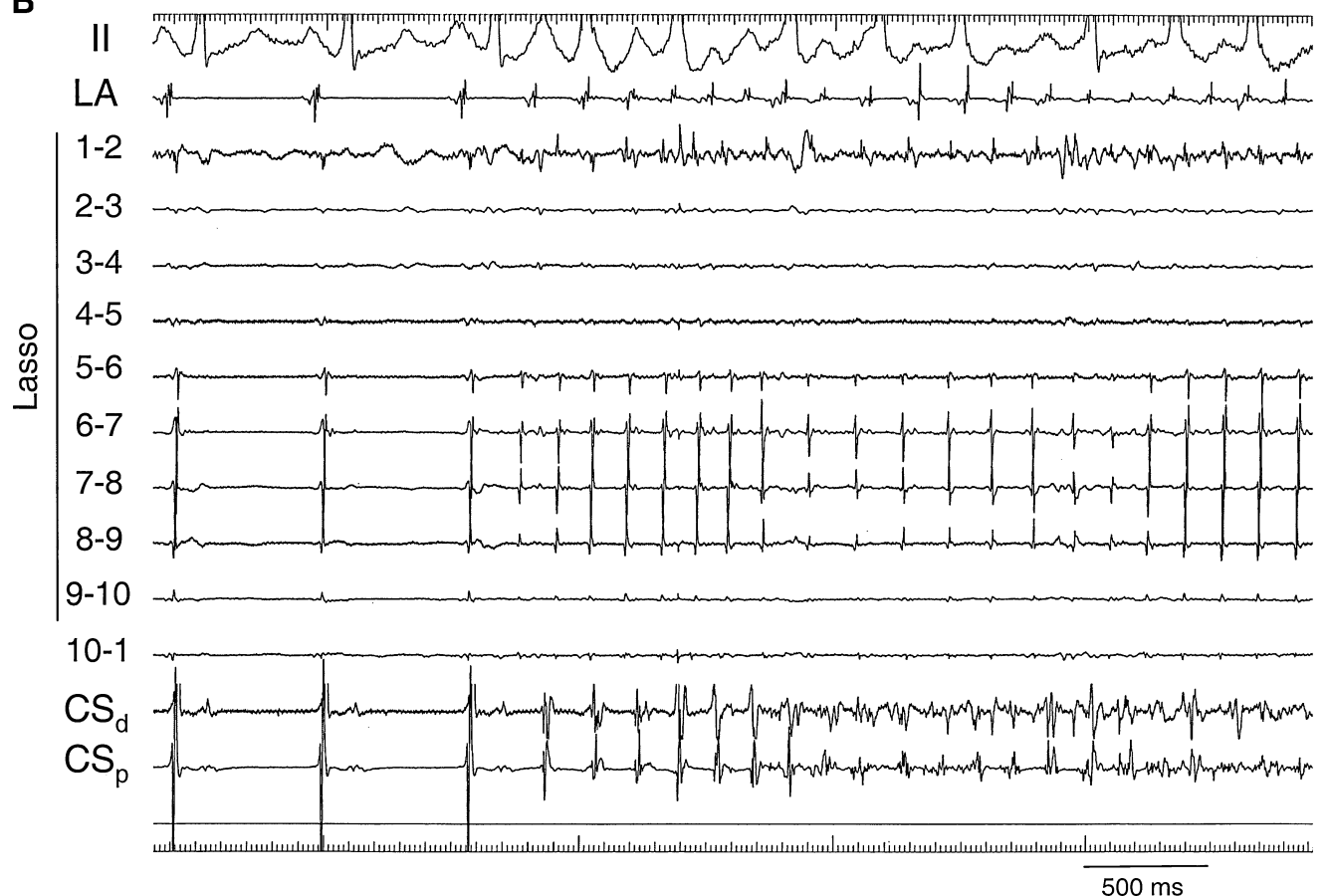

Figure 2. Immediate recurrence of atrial fibrillation (AF) triggered by a burst of pulmonary vein (PV) tachycardia, but not by a single premature depolarization. (Panel A) A premature PV depolarization with a coupling interval and prematurity index of $206 \mathrm{~ms}$ and 0.30 fails to trigger immediate recurrence of $A F$ after electrical cardioversion. The coupling interval was defined as the interval from the prior sinus depolarization to the onset of the premature depolarization (solid line). The prematurity index was defined as the ratio of the coupling interval to the preceding sinus cycle length (dashed line). (Panel B) In the same patient, a burst of PV tachycardia with a coupling interval and prematurity index of $192 \mathrm{~ms}$ and 0.33 , initiated an episode of immediate recurrence of AF. Catheter positions and abbreviations as in Figure 1. 
of the bursts of PV tachycardia were $246 \pm 67 \mathrm{~ms}$, and $0.30 \pm 0.11$, respectively.

The coupling interval and prematurity index of the PV depolarizations that did and did not trigger immediate recurrence of AF were $246 \pm 67 \mathrm{~ms}$ and $0.30 \pm 0.11$ vs $378 \pm 117 \mathrm{~ms}$ and $0.49 \pm 0.16$, respectively $(\mathrm{P}<0.01)$.

\section{PV Isolation}

Among 306 PVs, 293 (96\%) were successfully isolated by segmental ostial applications of radiofrequency energy. After PV isolation, there were no further episodes of immediate recurrence of AF.

\section{Main Findings}

\section{Discussion}

A prior study demonstrated that immediate recurrence of AF is consistently abolished by PV isolation, indicating that the PVs are a common source of the trigger for immediate recurrence of AF. The present study demonstrates that the trigger of immediate recurrence of $\mathrm{AF}$ always is a burst of PV tachycardia, as opposed to a single premature depolarization.

\section{Mechanisms of Immediate Recurrence of AF}

Compared to a single premature depolarization, a burst of PV tachycardia may facilitate immediate recurrence of AF in several ways. First, consecutive premature stimuli may progressively shorten the PV fascicular and left atrial effective refractory periods so that subsequent stimuli with shorter coupling intervals can conduct to the left atrium and trigger AF. Second, AF usually may be more readily induced by rapid atrial burst pacing than a single extrastimulus. Rapid atrial pacing may facilitate the induction of AF by creating multiple sites of functional block that serve as a substrate for reentry. It is possible that bursts of PV tachycardia have a similar effect on the atrium.

\section{Immediate Recurrence of AF After Spontaneous and Transthoracic Cardioversion}

Immediate recurrence of AF occurred less often after spontaneous conversion than after transthoracic cardioversion. A possible explanation for this finding is that the trigger for immediate recurrence of $\mathrm{AF}$ is the same type of $\mathrm{PV}$ tachycardia that plays a role in maintaining $\mathrm{AF}^{7}$ and that spontaneous conversion occurs only because PV tachycardia has become less frequent. Therefore, the immediate recurrence of AF may not have occurred as often because of the same reason that AF converted spontaneously. However, the higher prevalence of immediate recurrence of AF after transthoracic cardioversion may also be explained by a stimulatory effect of transthoracic shocks on the PVs. Additional studies are required to determine which one of these explanations is more likely.

\section{Clinical Significance of Immediate Recurrence of AF in Patients with AF}

In a prior study, immediate recurrence of $\mathrm{AF}$ that occurred during an electrophysiological procedure in patients with paroxysmal AF was consistently abolished by complete electrical isolation of the PVs. ${ }^{4}$ This finding suggests that immediate recurrence of AF is primarily triggered by premature depolarizations that originate from the PVs.

Intermittent PV tachycardias have been shown to play a critical role in the initiation and perpetuation of $\mathrm{AF}^{4,7,10}$ However, because the mechanism of $\mathrm{AF}$ is multifactorial, ${ }^{11} \mathrm{PV}$ isolation by itself does not always prevent AF. It would be helpful to be able to identify the patients who are most likely to benefit from a PV isolation procedure. A clinical variable that is predictive of a successful outcome after PV isolation is paroxysmal (as opposed to persistent) AF. ${ }^{9,12}$ The results of this study suggest that another clinical variable that may be helpful in selecting patients for PV isolation is an episode of immediate recurrence of AF after cardioversion.

\section{Previous Studies}

No prior studies have described the triggers of immediate recurrence of AF. A prior study in which immediate recurrence of $\mathrm{AF}$ was used to map arrhythmogenic foci within PVs suggested that immediate recurrence of $\mathrm{AF}$ was triggered by premature depolarizations from the PVs, but the characteristics of the triggers were not described. ${ }^{5}$

\section{Study Limitations}

PV recordings were available from only one $\mathrm{PV}$ at a time. Because there was no reliable method to predict which PV would be the source of immediate recurrence of $\mathrm{AF}$, the ring catheter often was positioned in a PV that was not the source of the immediate recurrence of $\mathrm{AF}$, and the trigger for immediate recurrence of AF could be characterized in only $19 \%$ of episodes of immediate recurrence of AF. The possibility that immediate recurrence of AF was triggered by a source outside the PVs cannot be excluded. However, this possibility is unlikely since immediate recurrence of AF was consistently abolished by PV isolation, as previously reported. ${ }^{4}$

\section{Conclusions}

In this study, all instances of immediate recurrence of AF were triggered by a burst of PV tachycardia, not simply a premature depolarization. An episode of immediate recurrence of $\mathrm{AF}$ 
after transthoracic or spontaneous cardioversion of AF may identify the patients who are most likely

\section{References}

1. Daoud EG, Hummel JD, Augostini R, et al. Effect of verapamil on immediate recurrence of atrial fibrillation. J Cardiovasc Electrophysiol 2000; 11:1231-1237.

2. Van Noord T, Van Gelder IC, Schoonderwoerd BA, et al. Immediate reinitiation of atrial fibrillation after electrical cardioversion predicts subsequent pharmacologic and electrical conversion to sinus rhythm and amiodarone. Am J Cardiol 2000; 86:1384-1385, A5.

3. Yu WC, Lin YK, Tai CT, et al. Early recurrence of atrial fibrillation after external cardioversion. PACE 1999; 22:1614-1619.

4. Oral H, Knight BP, Ozaydin M, et al. Segmental ostial ablation to isolate the pulmonary veins during atrial fibrillation: Feasibility and mechanistic insights. Circulation 2002; 106:1256-1262.

5. Lau CP, Tse HF, Ayers GM. Defibrillation-guided radiofrequency ablation of atrial fibrillation secondary to an atrial focus. J Am Coll Cardiol 1999; 33:1217-1226.

6. Timmermans C, Rodriguez LM, Smeets JL, et al. Immediate reinitiation of atrial fibrillation following internal atrial defibrillation. J Cardiovasc Electrophysiol 1998; 9:122-128. to benefit from a PV isolation procedure to prevent recurrent episodes of AF.

7. Oral H, Ozaydin M, Tada H, et al. Mechanistic significance of intermittent pulmonary vein tachycardia in patients with atrial fibrillation. J Cardiovasc Electrophysiol 2002; 13:645-650.

8. Haissaguerre M, Shah DC, Jais P, et al. Electrophysiological breakthroughs from the left atrium to the pulmonary veins. Circulation 2000; 102:2463-2465.

9. Oral H, Knight BP, Tada H, et al. Pulmonary vein isolation for paroxysmal and persistent atrial fibrillation. Circulation 2002; 105:10771081.

10. Haissaguerre M, Jais P, Shah DC, et al. Spontaneous initiation of atrial fibrillation by ectopic beats originating in the pulmonary veins. N Engl J Med 1998; 339:659-666.

11. Allessie MA, Boyden PA, Camm AJ, et al. Pathophysiology and prevention of atrial fibrillation. Circulation 2001; 103:769-777.

12. Pappone C, Oreto G, Rosanio S, et al. Atrial electroanatomic remodeling after circumferential radiofrequency pulmonary vein ablation: Efficacy of an anatomic approach in a large cohort of patients with atrial fibrillation. Circulation 2001; 104:2539-2544. 